\title{
el parbellám
}

\section{italiamo en Ia Expo-zO de Dsaka}

PROYECTO: Dr. Arq. Ing. T. y G. VALLE

Ing. S. BRUSA PASQUE

ESTRUCTURA: Prof. Ing. S. MUSMECI

\section{simopsis}

$149-20$

Consta de seis cuerpos, de sección rectangular de $14,5 \times 5 \mathrm{~m}$, con su eje inclinado $30^{\circ}$ respecto al plano horizontal; y de otros cuatro, de sección también rectangular, de $3 \times 4 \mathrm{~m}$ e inclinados, asi. mismo, $30^{\circ}$, pero en posición contraria a la de los precedentes. Los seis elementos primeros contienen las zonas destinadas a exhibición; y los cuatro últimos albergan: las comunicaciones verticales, las diferentes instalaciones, canalizaciones, tomas de aire, etc.

El organismo estructural creado es la expresión atrayente de una dimensión y de un espacio distintos y estimulantes de gran dinamismo y plasticidad.
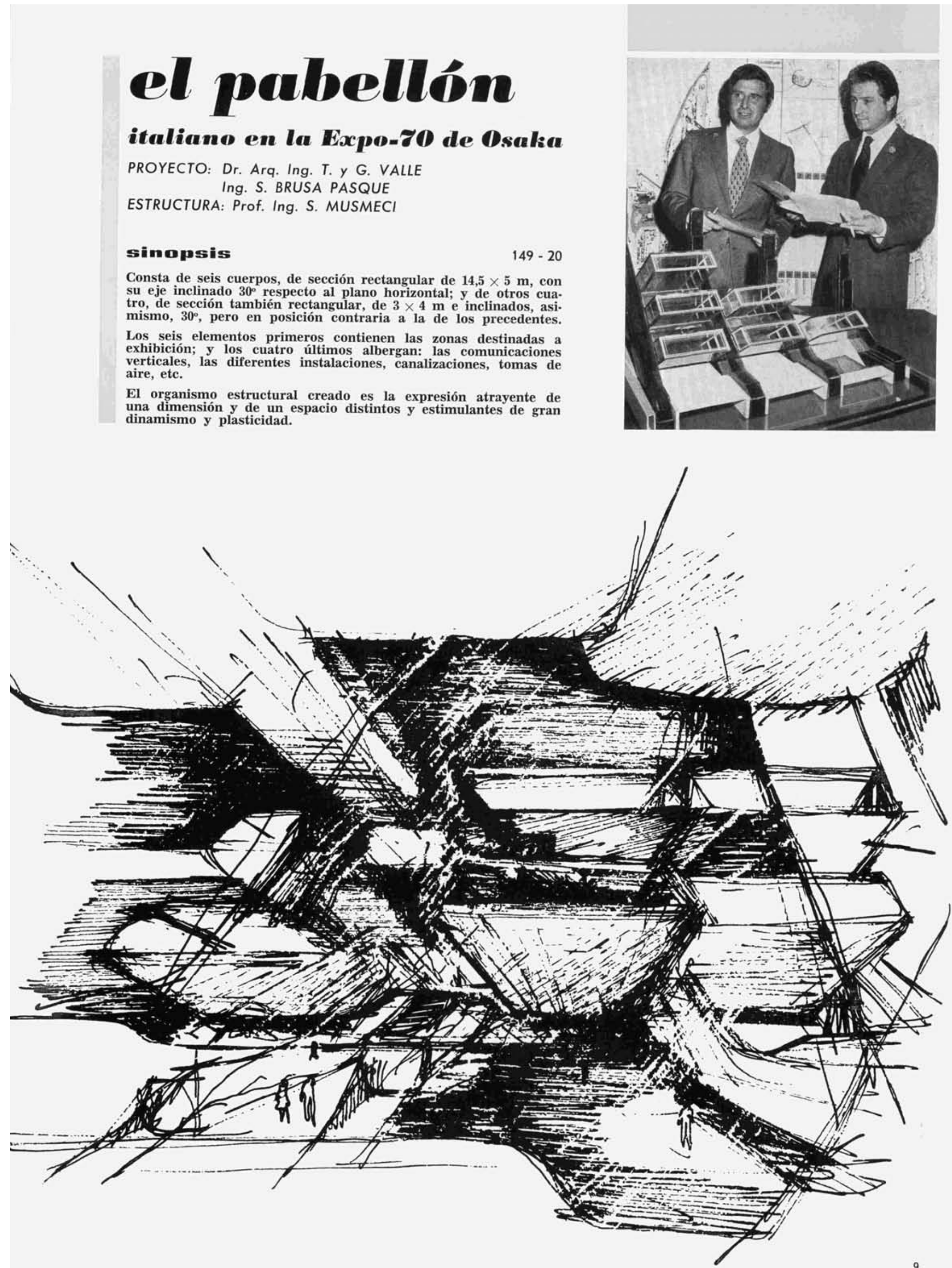


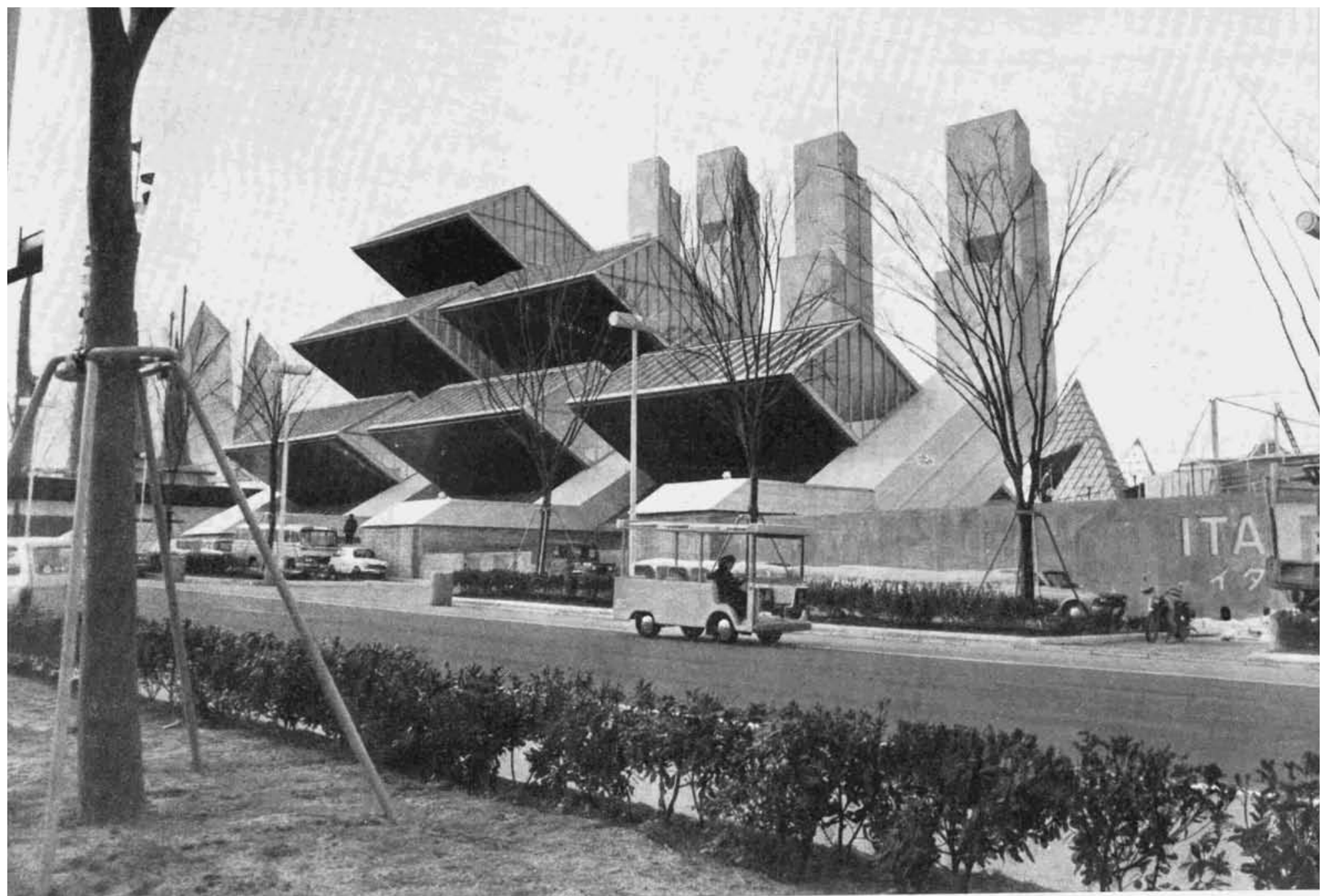

Fue diseñado partiendo de las siguientes premisas: mostrar una prestancia, a la altura de una Feria Internacional; invitar a reflexionar sobre el panorama económico y cultural, e insertar la dimensión espacial de su arquitectura en el marco de experimentaciones activas y de Artes Plásticas que se promueven con ocasión de tales certámenes.

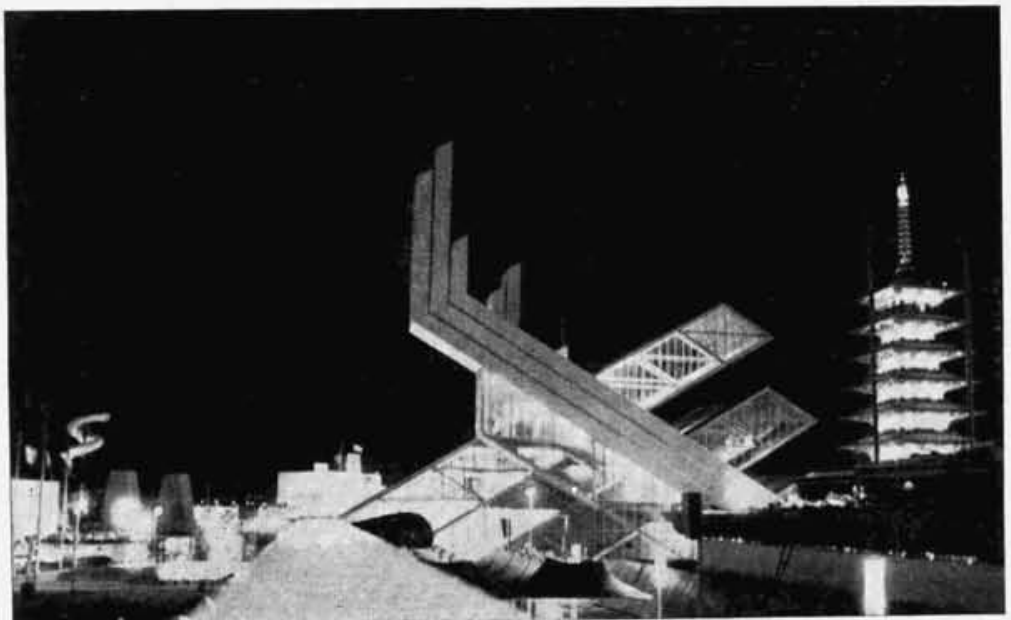

Fue construido en la Exposición Universal de Osaka 1970 que, como todos conocemos, ha sido motivo de confrontación de nuevos espacios y de nuevas dimensiones operativas.

Se alza sobre una parcela rectangular, limitada por dos calles, y se ha construido según el proyecto ganador del concurso convocado para su construcción, en el que participaron cerca de 200 arquitectos, ingenieros y artistas.

El Pabellón consta de: seis cuerpos o unidades constructivas, de sección rectangular de $14 \times 5 \mathrm{~m}$ y de longitud variable entre los 20 y $50 \mathrm{~m}$, con su eje inclinado $30^{\circ}$ respecto al plano horizontal; y de otros cuatro, de sección también rectan- 


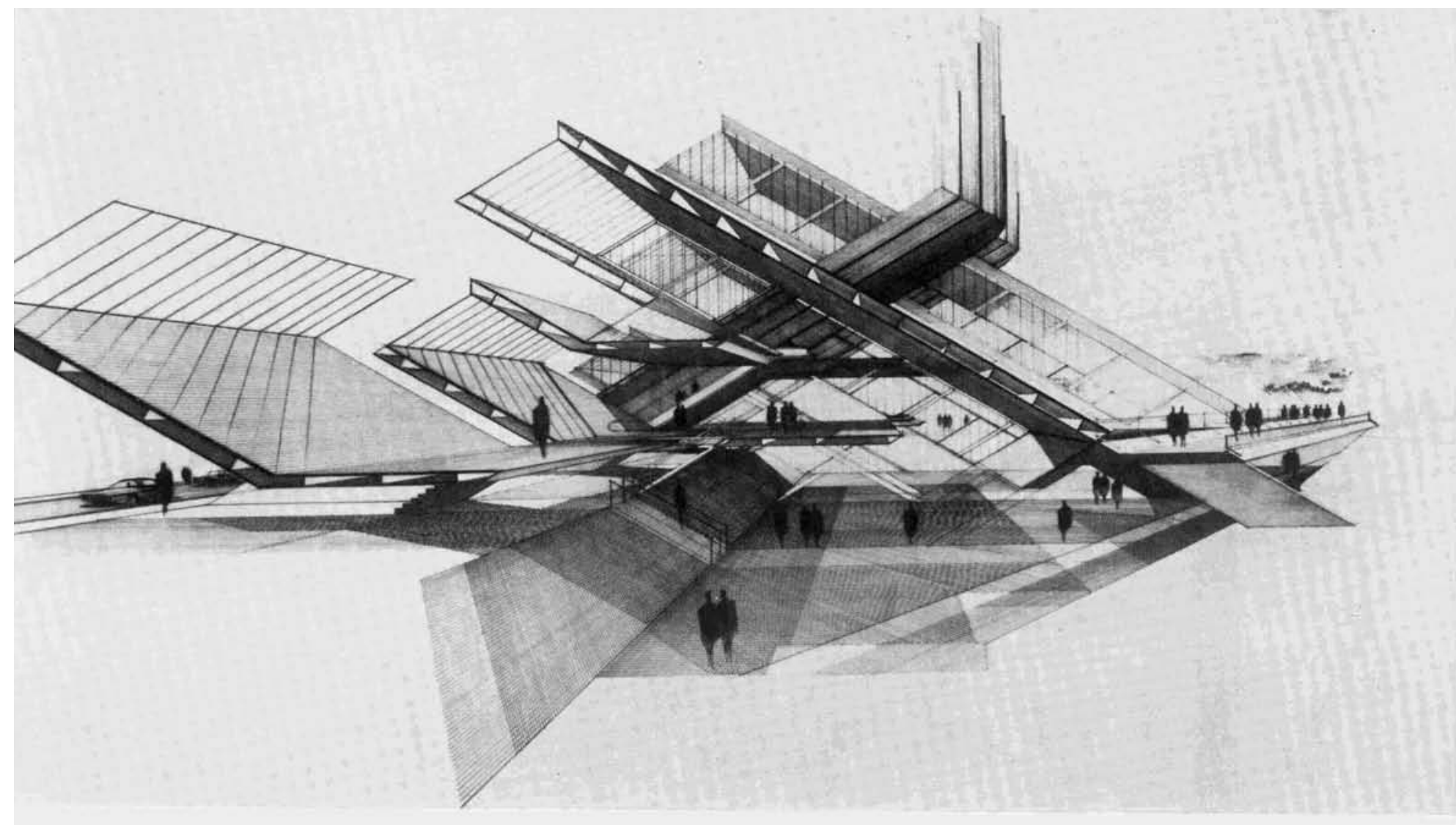

mpersmectivi an

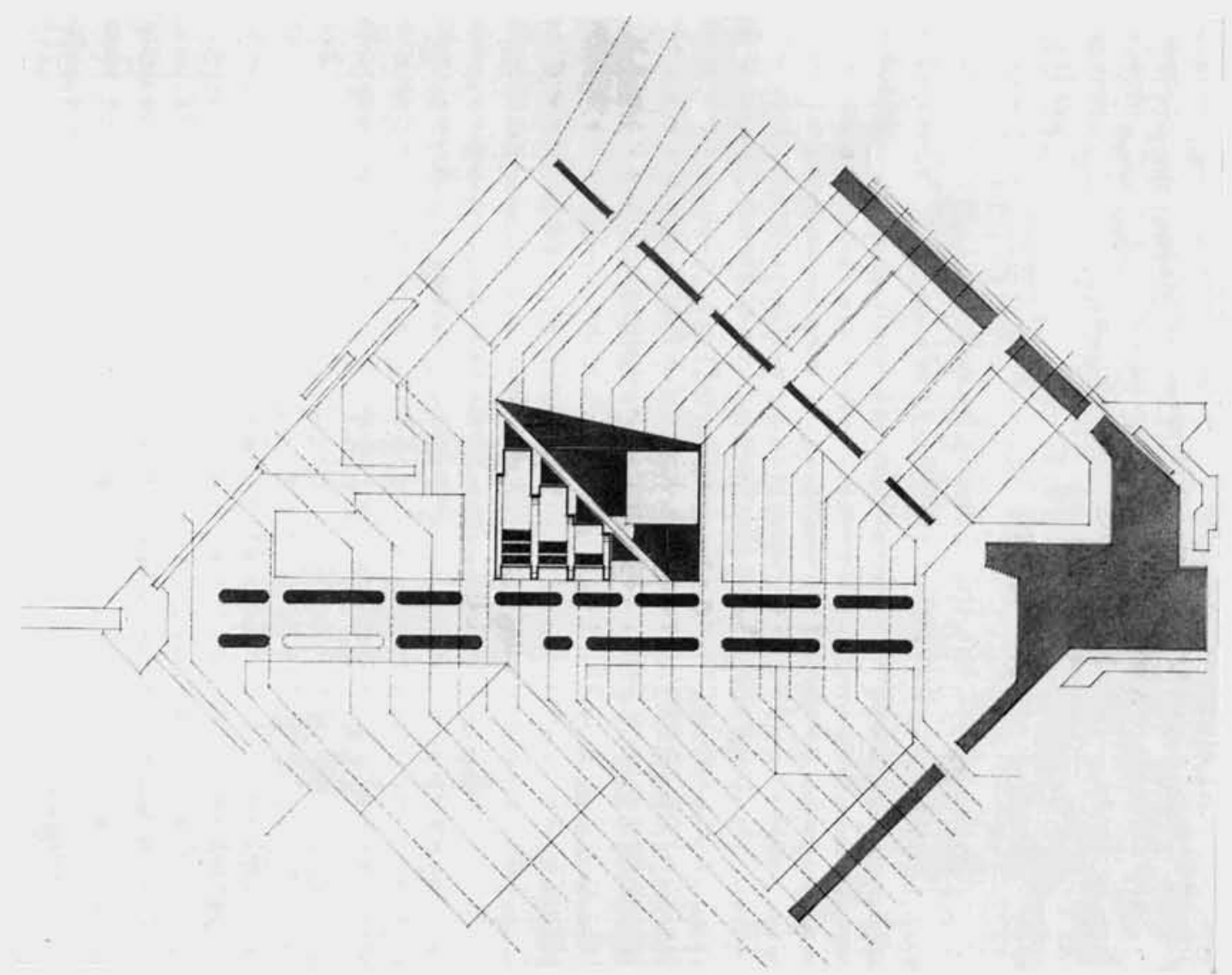




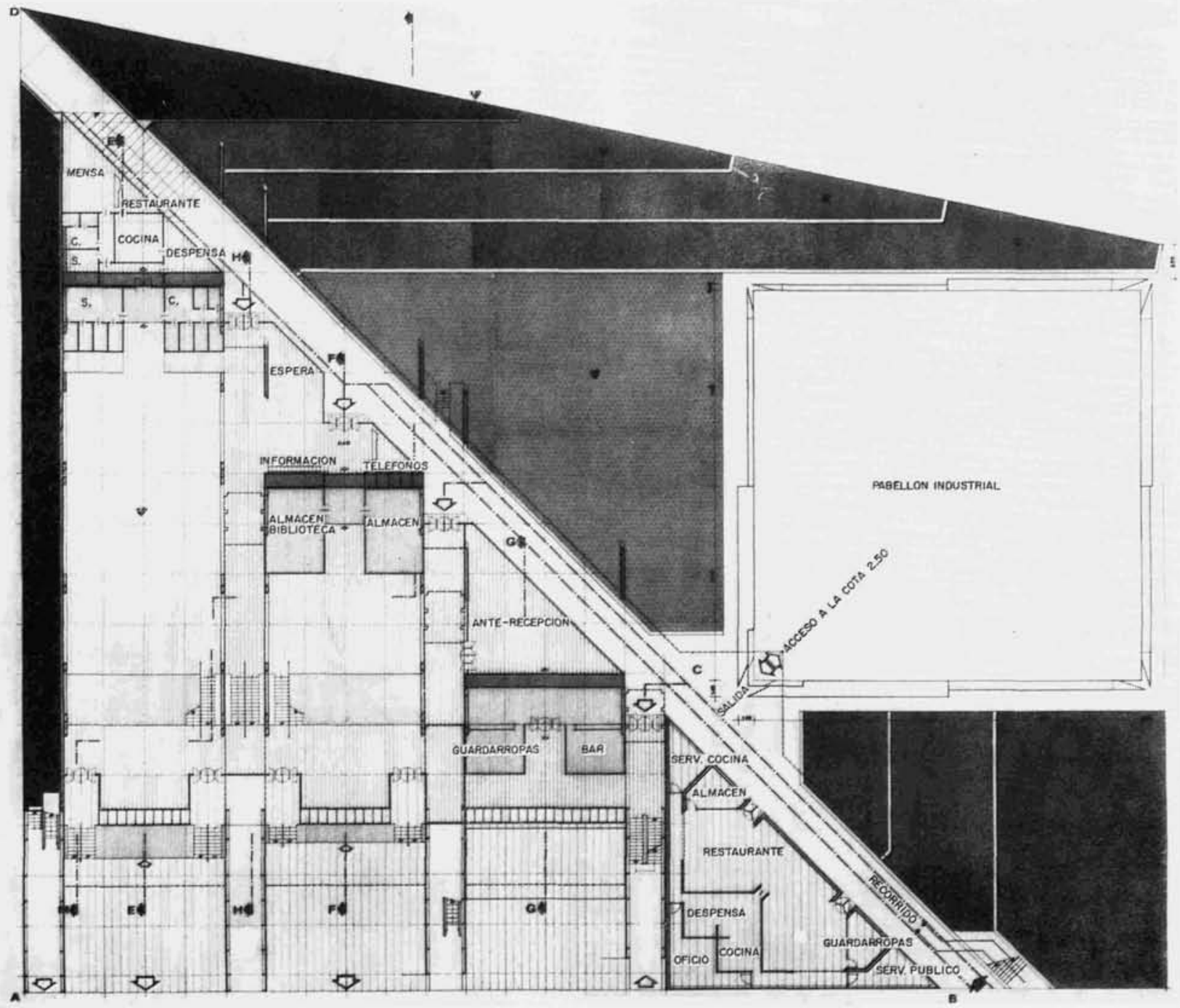

gular de $3 \times 4 \mathrm{~m}$ y de longitud variable entre los 35 y $55 \mathrm{~m}$ e inclinados asimismo $30^{\circ}$, pero en posición contraria a la de los precedentes.

Los seis elementos primeros contienen las zonas destinadas a exhibición; y los cuatro últimos albergan: las comunicaciones verticales, las diferentes instalaciones, canalizaciones, tomas de aire, etc. Ambos tipos de elementos aparecen contraponiéndose, apoyándose mutuamente, y creando, en su interior, un ambiente articulado.

Aunque desde el exterior se aprecian claramente las separaciones que establecen los nudos que forman desde dentro, la percepción del espacio interior es global.

Las zonas dedicadas a exhibición, cuya superficie alcanza un total de cerca de $4.000 \mathrm{~m}^{2}$, fueron articuladas a base de vuelos y de penetraciones recíprocas, bien en el sentido vertical o bien en el horizontal. Ocupan preferentemente la planta, sita al mismo nivel que el del terreno; y la del primer nivel, por el que se realiza el acceso del público. Los niveles intermedios, en las diversas cotas, permitieron una máxima flexibilidad para el montaje de la Exposición.

A la derecha, está situado un "colector», utilizado por el público que entra y sale; vía interior cubierta, que permite a la masa de visitantes, abreviando el recorrido, la visión de la totalidad del Pabellón, así como también el llegar a la Friday Plaza, punto de notable interés de la Exposición. 

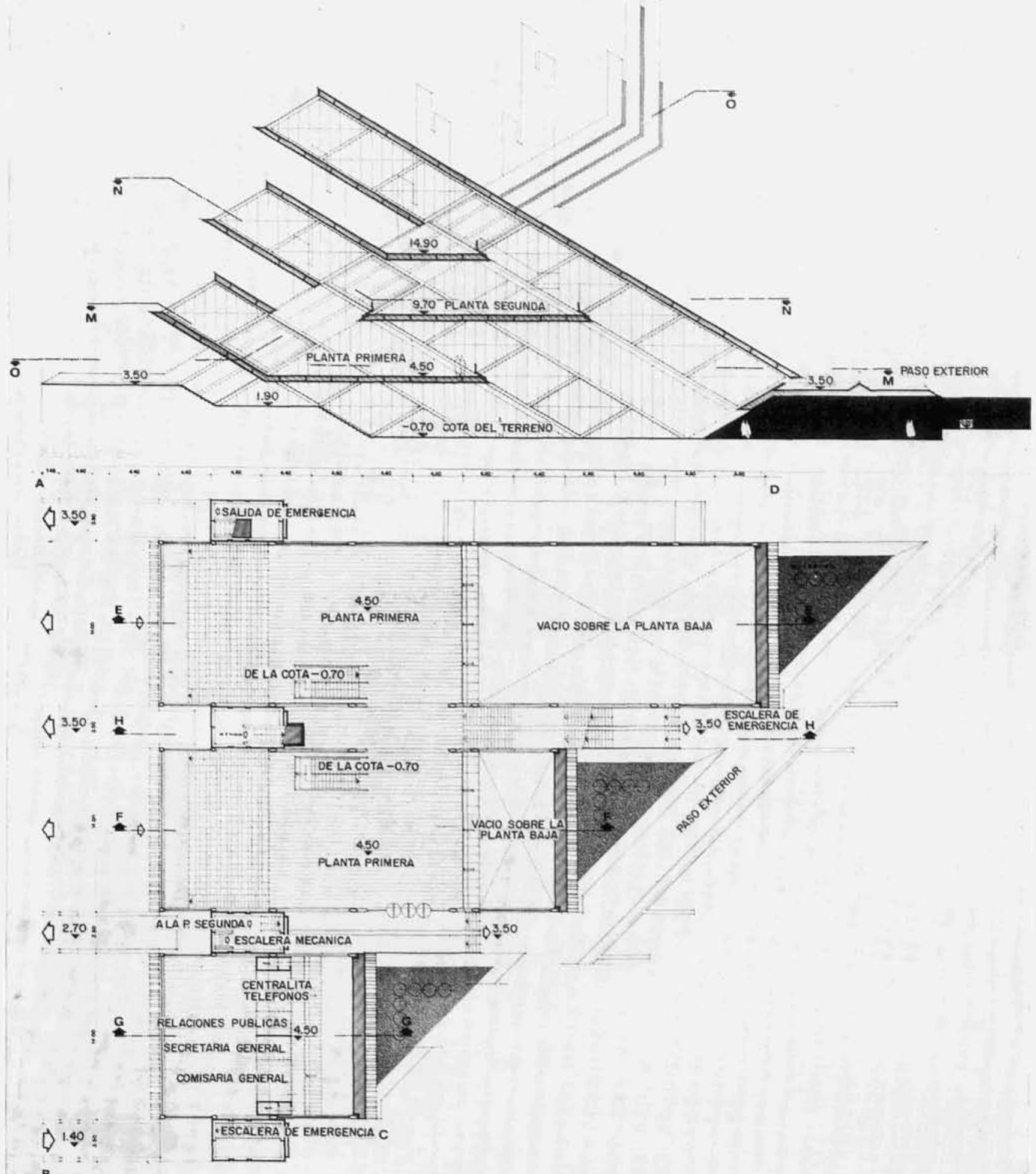

B 

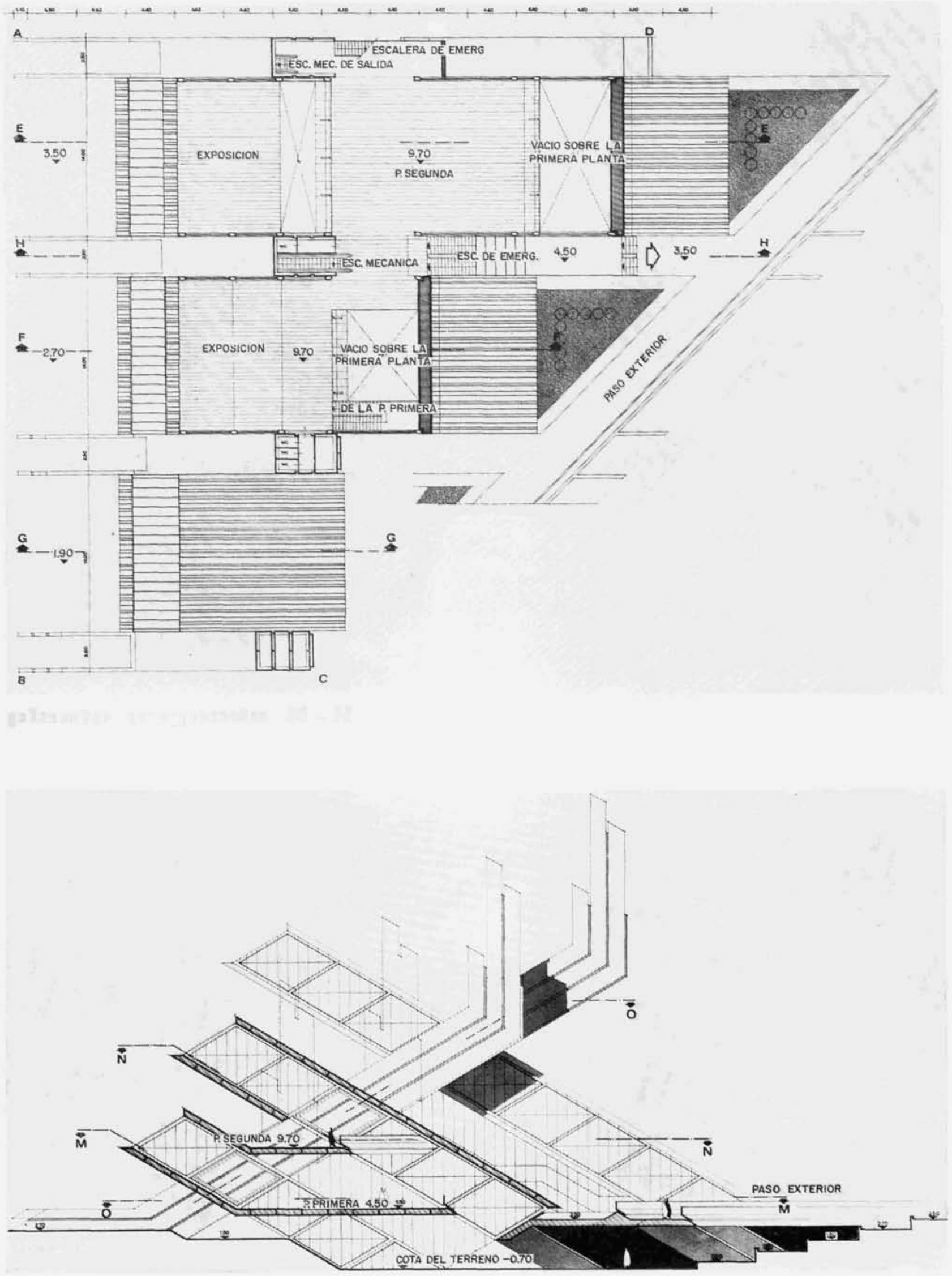

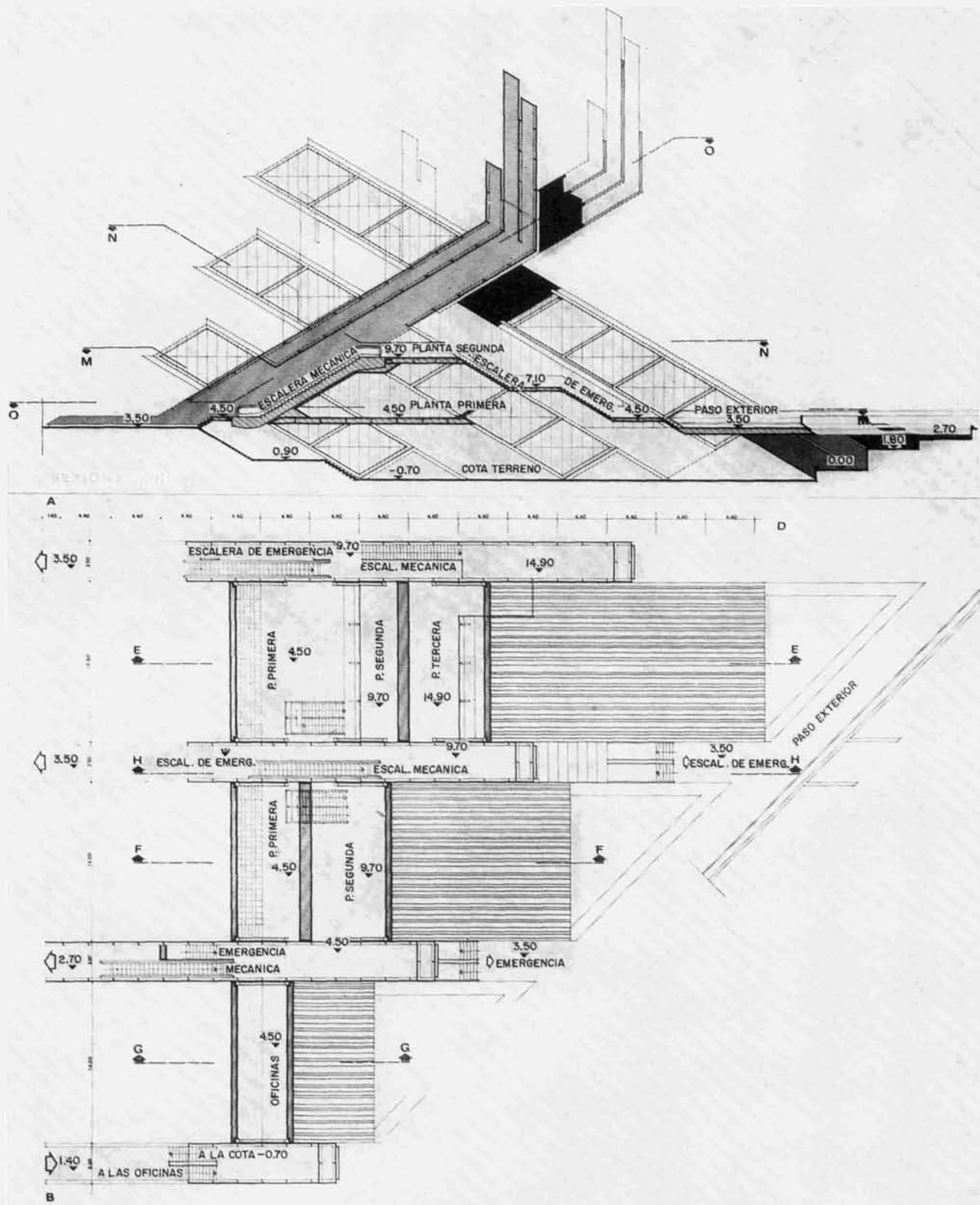


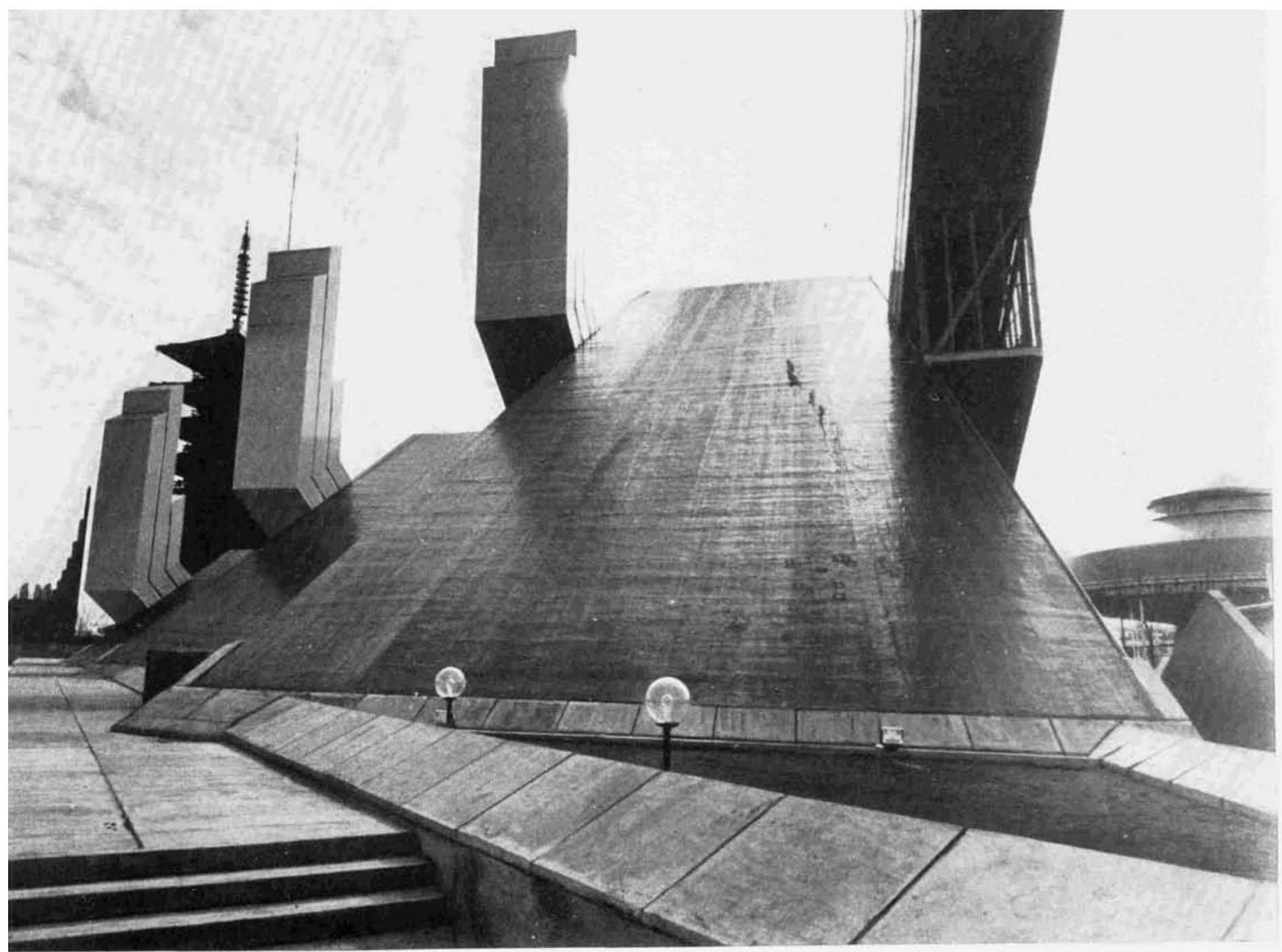

La estructura corpórea realizada en este Pabellón aparece fuertemente caracterizada, e imprime direccionalidad, dimensiones y límites al ámbito urbano. Dicha estructura fue además especialmente preparada para soportar los efectos de posibles sacudidas sísmicas. Mientras que en un edificio tradicional, las fuerzas son transmitidas a la cimentación a través de dos órdenes de elementos estructurales: los forjados horizontales, y los elementos verticales - con particulares cualidades de rigidez para recibir de los primeros las acciones debidas a las sacudidas-, en este Pabellón se prevé una transmisión directa de las acciones sísmicas al terreno a través de los planos inclinados.

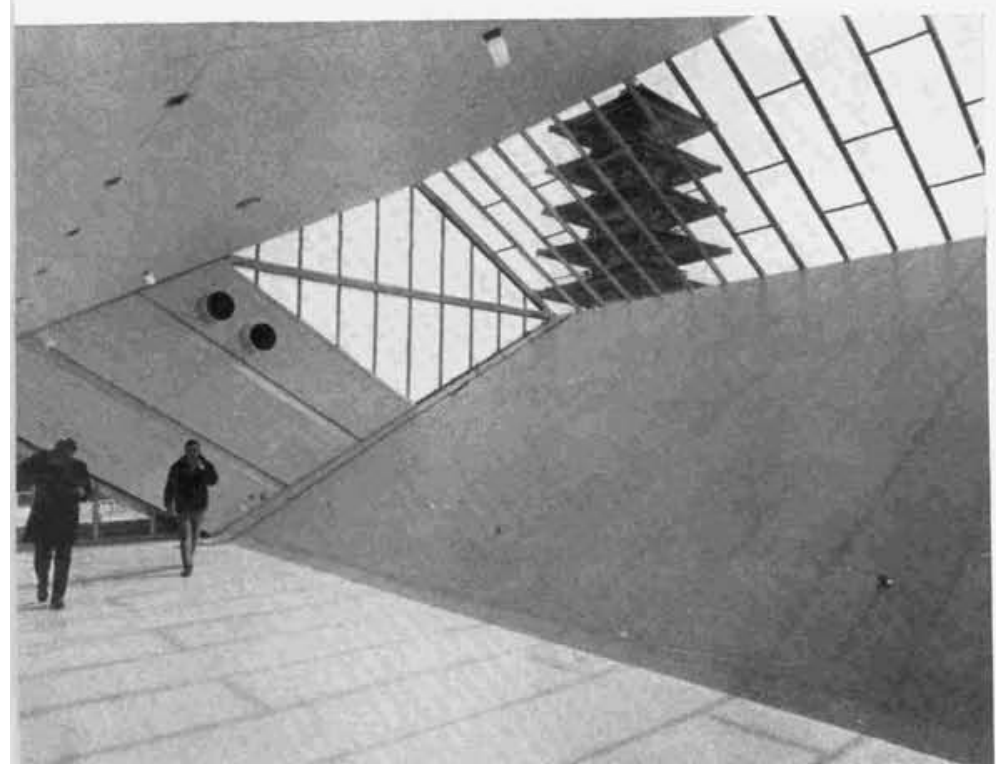

El sistema espacial entero planteado ha venido a plasmar y mostrar una dimensión, diversa y estimulante de un espacio, ligada a la dimensión del vivir civilizado e histórico; el mismo espacio, en su organización representativa y funcional, sirve como instrumento de conocimiento y factor activo para la vida del hombre.

Los cuerpos inclinados, visibles a la izquierda, contienen el material expositivo, dispuesto con criterios correspondientes al carácter "no tradicional» del Pabellón; las aberturas laterales, desarrolladas en altura, han hecho posible el disfrute de atractivos efectos conseguidos con la luz. 
El organismo estructural, que aparece claramente visible en todas sus partes, está constituido solamente por algunos elementos tipo, cada uno de ellos fuertemente caracterizado respecto a los otros, y todos con categoría para imponerse por su importancia formal y dimensional. Además, su posición en el espacio -deliberadamente contrapuestos- pone de manifiesto la diferenciación de funciones, que se desarrollan en su interior.

Todo ello confiere gran fuerza al significado estructural de las partes y del todo, ayuda a destacar este Pabellón sobre el «rumor de fondo" que siempre respira la atmósfera dinámica de una Exposición, y contribuye a señalizar su arquitectura como algo semánticamente articulado y cualificado.
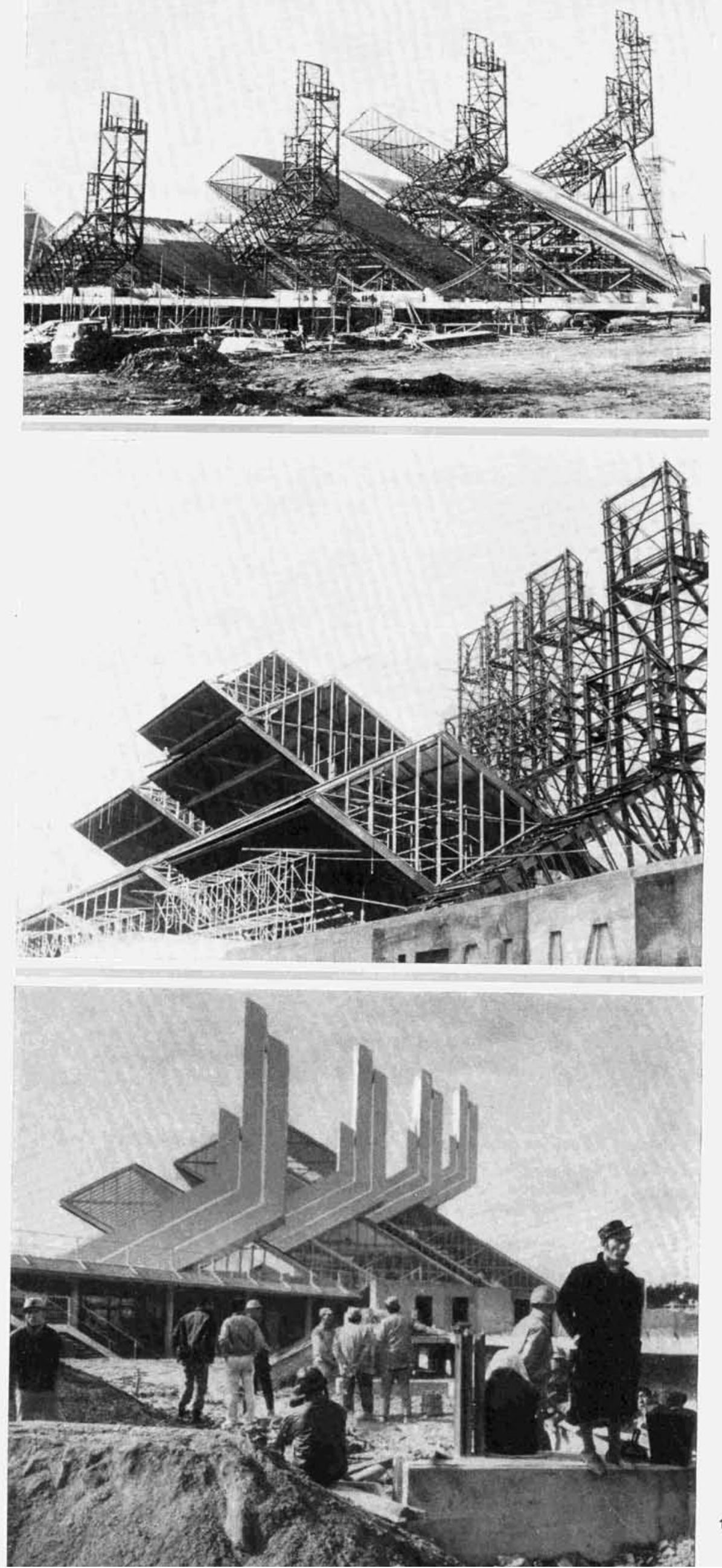


\section{Le paviuno italien de rexpo-70 à Osako}

Projet: Dr. Arch. Eng. T. \& G. Valle, ing. S. Brusa Pasque

Structure: Prof. ing. S. Musmeci

Il se compose de six corps, de section rectangulaire de $14, \times 5 \mathrm{~m}$, d'axe incliné de $30^{\circ}$ par rapport au plan horizontal, et de quatre autres de section également rectangulaire, de $3 \times 4 \mathrm{~m}$, également inclinés de $30^{\circ}$, mais en sens contraire.

Les six premiers éléments comprennent les zones d'exposition proprement dite, et les quatre autres sont réservés aux communications verticales, aux différentes installations, canalisations, prises d'air, etc.

La structure ainsi créée est l'expression remarquable d'une dimension et d'un espace différents et stimulants, d'un grand dynamisme et d'une pure plasticité.

\section{The Italian Paviaion of ExPO-70, at Osake, Japan}

Project: Dr. Arch. Eng. T. \& G. Valle, S. Brusa Pasque, engineer

Structure: Prof. Eng. S. Musmeci

This pavillion has six elements, each on a $14.5 \times 5 \mathrm{~m}$ rectangular site, and the axis inclined $30^{\circ}$ to the horizontal; and a further four, also rectangular, on a $4 \times 3 \mathrm{~m}$ base, similarly inclined, but in the opposite sense to the first six.

The first six are devoted to exhibitions zones, and the latter four contain vertical communications, installations, conduits, air supplies, etc.

This structural system is an attractive expression of novel space and dimensional arrangements, and conveys a highly dynamic and plastic impression.

\section{Den Italiamische Pavillom anf der EXPO-70 in Osaka}

Projekt: Dr. Arch. Ing. T. \& G. Valle, Ing. S. Brusa Pasque

Struktur: Prof. Ing. S. Musmeci

Er besteht aus sechs, im Schnitt rechteckigen Körpern in den Abmessungen $14,5 \times 5 \mathrm{~m}$, deren Achse in Bezug auf die horizontale Ebene um $30^{\circ}$ geneigt ist, sowie weiteren vier Körpern mit ebenfalls rechteckigem Querschnitt $3 \times 4 \mathrm{~m}$, die gleichfalls, jedoch in entgegengesetzter Richtung um $30^{\circ}$ geneigt sind.

In den sechs ersteren Elementen sind die Ausstellungsräume untergebracht, die letzteren vier umfassen: die vertikalen Verbindungswege, dlie verschiedenen Installationen, Kanalisierungen, Entlüfturgen u.s.w.

Die so entstandene organische Struktur ist attraktiver Ausdruck unterschiedlicher und stimulierender Darstellungen von Dimension und Raum und zeigt grosse Dynamik und Plastizität. 